\title{
Rule Based Adaptation: Literature Review
}

\author{
Lauma Jokste, Janis Grabis \\ Information Technology Institute, Riga Technical University, Kalku 1, Riga, Latvia, grabis@ rtu.lv
}

\begin{abstract}
Rule based adaptive systems are growing in popularity and rules have been considered as an effective and elastic way to adapt systems. A rule based approach allows transparent monitoring of performed adaptation actions and gives an important advantage of easily modifiable adaptation process. The goal of this paper is to summarize literature review on rule based adaptation systems. The emphasis is put on rule types, semantics used for defining rules and measurement of effectiveness and correctness of rule based adaptation systems. The literature review has been done following a systematic approach consisting of three steps: planning, reviewing and analysis. Targeted research questions have been used to guide the review process. The review results are to be used for conducting further research in the area of rule based context-aware adaptive systems. This paper accents the potential of using rules as means to perform adaptive actions in enterprise applications taking into account contextual factors as well as points challenges, difficulties and open issues for planning, developing, implementing and running of such systems.
\end{abstract}

Keywords: rules, rule based adaptation.

\section{INTRODUCTION}

Rule based adaptation systems have become very popular in last decades. In rule based adaptation systems adaptation actions are performed in form of rules - statements that tells system what is or what is not allowed or what should be done in particular situation. Rules have been considered as effective means for performing adaptive actions in different systems. The rule based adaptation systems have advantages of readability, transparency, elegance and they are easy modifiable by simply adding, removing or modifying rules [1]. Tran et al. in [2] emphasize that the most important advantage of using rules are the fact that rules can be managed and controlled by humans. Rubart in [3] also considers the ability of managing rules by both - humans and system components as an advantage.

The goal of this literature review is to explore the existing scientific literature on rule based adaptation systems. The sub goals are to explore the potential of using rules as means to perform adaptive actions in enterprise applications as well as to identify the shortcomings and open challenges for such systems.

The rest of the paper is structured as follows: section II describes the literature review methodology and research questions. Section III shows the results of the conducted literature review structured according to research questions defined in section II. Section IV gives some conclusions and observations about the analyzed literature.

\section{MATERIALS AND METHODS}

A literature review has been divided into three stages: planning the review, conducting the review and reporting the review [4]. In order to initiate the literature review, a literature review protocol has been developed (see figure 1). The review protocol ensures systematic and iterative approach for developing literature review by defining how each step of the review will be performed [5].

Following research questions (RQ) have been defined in order to guide analysis of literature:

- RQ1: For what kind of software entities rules have been applied?

- RQ2: What types and semantic forms of rules have been used?

- RQ3: Application domains where rule based systems are used?

- RQ4: What kind of rule performance measures are used?

- RQ5: What are open challenges and potential solutions?

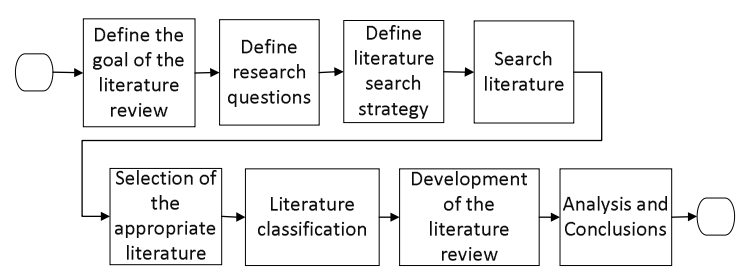

Fig. 1. Literature review protocol.

The literature search strategy includes following conditions:

- Research papers are searched in scientific data bases accessible for the authors: IEEE, ScienceDirect, The ACM Digital Library, Springer Link.

- Key words used for the search process: adaptation rules, rule based adaptive systems, rule based systems, event-action rules. 
- Publication years are limited from 2000 2017.

- Research paper types are limited to: research papers, conference proceedings, periodicals, doctoral dissertations, technical reports and books.

In total 112 papers were found from which 45 papers were selected for further analysis and inclusion into literature review.

The literature review results will be used as a base for further research in the area of rule based contextaware adaptive systems, thereby research questions have been defined based upon the specification of the approach to be developed.

\section{RESULTS AND DISCUSSION}

The literature review has been structured based on the research questions defined in section II. Due to the space limitations, only the most important results are described and summarized in this paper.

A. RQ1: For what kind of software entities rules have been applied

Rule based adaptation can be applicable for different type of systems and different adaptable software entities. One of the most popular adaptable entities is users interface. López-Jaquero et al. in [6] emphasize that adaptation rules allow to evolve the users interface in accordance with the evolvement of systems' users.

Rule based adaptive systems mostly focus on one particular type of software entities, e.g. processes, graphical users interface components etc. We assume that systems consist of standardized objects, such as classes, forms, procedures etc. These objects are referred as software entities and each of them can be context dependent. Rules can be defined for different kind of software entities. Following types of adaptable software entities in adaptation systems were identified in current investigations:

- Process adaptation - adaptable entities are processes or process schemas [7] - [8].

- Workflow and service flow adaptation workflow and service flow technologies are widely used in complex processes in web applications to reach the flexibility. Workflow as an adaptation entity is described in [9] and service flow is described in [10].

- Content adaptation - adaptable entities are content parts such as pictures, text, command buttons etc. [11] - [12].

- GUI/AUI adaptation - adaptable entities are graphical users interface parts or audio interface parts. Often adaptation in this level is performed for complex GUI, especially in heterogeneous systems [13] - [14] and for systems used by users with disabilities [15] [16].

- Software configuration adaptation software as a whole can be configured based upon the received context information [12].

- Features adaptation - features can be both functional and technical components of the software, e.g. browser, desktop, Bluetooth [1].

The wide range of different software components adaptation performed in a form of rules proves the flexibility and usefulness of the rules.

B. RQ2: What types and semantic form of rules have been used?

Some papers discuss the rule based adaptation but do not specify the semantic form of rules used in proposed approaches. The most popular semantic form of rules used in rule based adaptation systems is Event-Condition-Action (ECA) rules with a typical form of IF-THEN-(ELSE). Some authors have used a derivate form of ECA rules, e.g. Muller et al. in [9] uses form WHEN (event) WITH (condition) THEN (control action) VALID-TIME (time period), including condition about time period in which this control action is valid. The time parameter has been taken into account also in [7] and [17]. In [15] an extra parameter - description is used for better understandability of the rule. In [18] RuleML is used as the most suitable language for medicine related adaptive systems. This language ensures equally good readability for both - humans and machines. Some authors use their own rules languages or have adjusted existing rule languages for their specific needs. In [19] a combination of 4 languages is used for event based software components adaptation. One of the ways how to describe rule semantic and rules language components is a rules metamodel [6]. The identified semantic forms of rules used in rule based adaptation systems are summarized in Table I.

Table I

Identified semantic forms of rules used in rule based adaptation systems

\begin{tabular}{|l|l|l|}
\hline Semantic form & Description & References \\
\hline $\begin{array}{l}\text { Event- } \\
\text { Condition- } \\
\text { Action rule }\end{array}$ & $\begin{array}{l}\text { Defined in form IF-THEN- } \\
\text { (ELSE). }\end{array}$ & $\begin{array}{l}{[7],[9]-} \\
{[10],[16],} \\
{[20]}\end{array}$ \\
\hline $\begin{array}{l}\text { Association } \\
\text { rules }\end{array}$ & $\begin{array}{l}\text { Are used to describe the } \\
\text { relationships between } \\
\text { elements or variables }\end{array}$ & {$[21]$} \\
\hline $\begin{array}{l}\text { RuleML based } \\
\text { behavioral } \\
\text { rules }\end{array}$ & $\begin{array}{l}\text { Language contains elements, } \\
\text { individual constants, data } \\
\text { values and complex } \\
\text { expressions. }\end{array}$ & {$[18],[22]$} \\
\hline
\end{tabular}

Rules in rule based adaptation systems also can be classified based on the adaptation entity and situations when adaptation actions are performed. Such classification is summarized in table II. 
Table II

Identified rule types used in rule based adaptation systems

\begin{tabular}{|c|c|c|}
\hline Rule type & Description & References \\
\hline $\begin{array}{l}\text { Content analysis } \\
\text { rules }\end{array}$ & $\begin{array}{l}\text { Used to gather the information about objects, such as the size of command button can be calculated } \\
\text { based on the number of characters used in command button. }\end{array}$ & {$[11],[15]$} \\
\hline $\begin{array}{l}\text { Content } \\
\text { adaptation rules }\end{array}$ & $\begin{array}{l}\text { Derived from the facts about the user. Content adaptation rules can be distinguished by the } \\
\text { adaptable content type - text changes, pictures changes etc. }\end{array}$ & {$[11]$} \\
\hline Corrective rules & $\begin{array}{l}\text { Used to adapt the application in case if applications current configuration is not viable in current } \\
\text { conditions. }\end{array}$ & {$[23]$} \\
\hline Enhancing rules & $\begin{array}{l}\text { Used to improve the existing activities which may work anyway but might work better. It can be } \\
\text { achieved by changing the non-functional properties of the activity or by providing a new } \\
\text { functionalities }\end{array}$ & [23] \\
\hline Fuzzy rules & $\begin{array}{l}\text { These rules are able to choose the most appropriate parameters based on heuristics. Fuzzy rules can } \\
\text { contain IF-THEN structure. }\end{array}$ & {$[24]-[25]$} \\
\hline Integration rules & $\begin{array}{l}\text { Used to represent the business logic for integrating the components in a declarative manner at a high } \\
\text { level of abstraction. They can be expressed in a form of ECA rules. }\end{array}$ & [19] \\
\hline Monitor rules & $\begin{array}{l}\text { Used to verify the service oriented systems behavior, systems services quality and context } \\
\text { information that affects the services used in the system and the system itself. }\end{array}$ & {$[17],[26]$} \\
\hline Production rules & $\begin{array}{l}\text { These rules are more appropriate for context-aware adaptation systems - rules are suitable for } \\
\text { reacting to newly received context information. }\end{array}$ & {$[12]$} \\
\hline Matching rules & $\begin{array}{l}\text { These rules are used in matching processes and they consists of several parts - pattern, action, } \\
\text { relevance function and an optional check function. }\end{array}$ & {$[8]$} \\
\hline
\end{tabular}

\section{RQ3: Application domains where rule based} systems are used?

Rule based adaptation systems are widely used in different area domains what reaffirms the benefit of such systems. In the literature reviewed, rules have been applied for the following domains: travel scenarios development systems [10], [23], different web applications, e.g. online auction site [27], medicine related systems [9], [18], smart building systems [28], specifically adjustable systems for people with disabilities [15], [29], mobile device applications [30] and others.

The usage of rules is not limited to adaptive systems. Rules are also used in data cleaning systems [31], software configuration management systems [32] and expert systems [25].

D. RQ4: What kind of rules performance measures are used?

In order to evaluate the quality of rules and the quality of the performed adaptive actions, several measures have been identified in analyzed literature:

- Confidence value has been calculated based upon collected users' feedback about the executed rule. Each time when positive feedback is fixed, rule's confidence value is increased. In the case of negative feedback, a new appropriate rule can be generated or other more appropriate rule from the existing rule set can be chosen and its confidence value is increased [20].

- Rule Weight [35], and Priority [34] measures can be used when several rules fit for one context situation - then a rule with the highest weight or priority can be executed.
Check function is used when the rule is executed. It allows to evaluate the quality of executed adaptation action initialized by the rule [8].

- Similarity degree [35] and Average Fitness Value [36] are used to measure the quality of automatically generated adaptation rules.

- Case based reasoning cycle can be used to evaluate the rules quality and improve the rules by searching similar rules from which new rules can be generated [33].

- Executed adaptation actions initialized by the rules can change systems metrics, e.g. response time, process execution time etc. These system metrics can be used to evaluate the quality of the executed adaptation rules [34].

- Users' preference can be fixed during the execution of recommended adaptive action - if user choose to execute the recommended action or prefers to execute another action [1].

Most of the Rule based adaptation systems use one particular approach for evaluating the quality of the adaptation rules or executed adaptation actions, thought combination of different approaches and rules quality measurements can ensure more accurate evaluation process and results.

\section{E. RQ5: What are the open challenges and potential solutions?}

Despite the fact that rule based adaptation systems are popular and they provide many advantages, there are also a number of limitations. For instance, Zhao [33] emphasizes that adaptation rules are often defined in advance with insufficient knowledge about executable actions what leads to poor performance of the rules when the system is in dynamic environment does not behave as expected. Following challenges of using rules have been identified:

- More than one rule fits one context situation: potential solutions are (1) to assign a static of 
dynamic priority to rules and to execute the rule with the highest priority [23], [34]; (2) rules can be executed in non-deterministic order [23]; (3) if possible, rules can be combined and executed simultaneously [34].

- A set of rules contains rules with equal IF part and different THEN part. Such rules are called conflicting rules [24]. Throughput can be taken into account and a rule with the highest throughput can be executed. In [20] conflicting rules can be clustered into one cluster and ranked according to their usage frequency. In [9] such problem can be solved by combining the executable actions, but in most cases there will be incompatibility, so there are no method for solving this problem completely.

- In an automatic rules generation process, a large number of rules can be generated. The challenge is to extract worthy rules. It can be solved by a two-step solution - in the first step duplicate rules can be identified. In the second step those who have equal IF part and different THEN part can be processed [20] as described previously.

- During the lifetime of adaptive system, a large amount of adaptation rules can gradually arise. To address this concern, Virgilio et al. [12] defines the optimization strategy based on a rule clustering technique. Rules clustering in clusters based on the type of affected context allows to manage the rules and to apply an effective rules search process.

Existing literature proves that open challenges still exists for using rules in adaptive systems, thereby there is a potential for improvement of such systems and approaches.

\section{CONCLUSIONS}

This paper presents the shortened form of the results of the literature review on rule based adaptation systems. The literature review results are divided into research questions and each research question leads to some insightful observations and conclusions.

The literature review highlight popularity and broad applicability of rules for adaptation of systems. Rules are used to adapt software entities of different kind, type and granularity level what indicates that rules are flexible and uniquely means how to perform adaptive actions. Nevertheless, some shortcoming and open challenges of using rules were identified during the literature analysis.
The results of the literature research summarize important information about the adaptation rules and rule based adaptation systems and this information will be further used to develop a new approach for adaption of context-aware software entities using rules.

\section{ACKNOWLEDGEMENTS}

The research leading to these results has received funding from the research project "Competence Centre of Information and Communication Technologies" of EU Structural funds, contract No. 1.2.1.1/16/A/007 signed between IT Competence Centre and Central Finance and Contracting Agency, Research No. 1.6 "Development of Software Adaptation Algorithms and Module Based on Context Information Extracted from Users Action Logs".

\section{REFERENCES}

[1] T. Zhao, H. Zhao, W. Zhang, and Z. Jin. User Preference Based Autonomic Generation of Self-Adaptive Rules, Internetware 2014 Proceedings of the $6^{\text {th }}$ Asia-Pacific Symposium on Internetware, pp. 25-34, 2014.

[2] T. Tran, P. Cimiano, A. Ankolekar, Rules for an Ontologybased Approach to Adaptation, Proceedings of the 1st International Workshop on Semantic Media Adaptation and Personalization. 2006.

[3] J. Rubart, Semantic Adaptation of Business Information Systems using Human-Centered Business Rule Engines, Proceedings of $10^{\text {th }}$ International Conference on Semantic Computing, pp. 187-193, 2016

[4] B. Kitchenham, Procedures for Performing Systematic Reviews. Joint Technical Review, 2004.

[5] A. Kofod-Petersen, How to do a structured literature review, Technical Report, 2014.

[6] V. López-Jaquero, F. Montero, F. Real, Designing User Interface Adaptation Rules with T:XML, Proceedings of the $14^{\text {th }}$ International conference on Intelligent user interfaces, pp.383-388, 2009.

[7] S. Yoo, Y. Roh, I. Song, J.H. Jeon, M.H. Kim, H.S. Kim, J. H.Son, Y. S. Paik, J.H. Han, H. K. Jang, Rule-based Dynamic Business Process Modification and Adaptation, Proceedings of International Conference on Information Networking, 2008.

[8] E. Peukert, J. Eberius, E.Rahm, Rule-based Construction of Matching Processes, Proceedings of the CIKM (Poster), pp. 2421-2424, 2011.

[9] R. Muller, U. Greiner and E.Rahm, AGENTWORK: a workflow system supporting rule-based workflow adaptation, Data and Knowledge Engineering, vol. 51, issue 2, pp. 223256, 2004.

[10] C. Ma, T.Wu, S. Wu, A Rule-based Approach for Dynamic Adaptation of Service Flow, Proceedings of 2012 5th IEEE International Conference on Service-Oriented Computing and Applications (SOCA), 2012.

[11] J. He, T. Gao, W. Hao, I.L. Yen, and F. Bastani, A Flexible Content Adaptation System Using a Rule-Based Approach, Transactions on Knowledge and Data Engineering, vol.19, no 1., pp.127-140, 2007.

[12] R. De Virgilio, R. Torlone, G.J. Houben, Rule-based Adaptation of Web Information Systems, WWW Journal, Publ. Springer Netherlands, 2007.

[13] P. Paskalev, I. Serafimova, Rule Based Framework for Intelligent GUI Adaptation, Proceedings of the $12^{\text {th }}$ International Conference on Computer Systems and Technologies, pp.101-108, 2011.

[14] P. Paskalev, Rule based GUI modification and adaptation, Proceedings of the International Conference on Computer Systems and Technologies and Workshop for $\mathrm{PhD}$ Students in Computing, article no. 93, 2009. 
Environment. Technology. Resources, Rezekne, Latvia Proceedings of the $11^{\text {th }}$ International Scientific and Practical Conference. Volume II, 42-46

[15] R. Miñón, F. Paternò, M. Arrue, An Environment for Designing and Sharing Adaptation Rules for Accessible Applications, Proceedings of the $5^{\text {th }}$ ACM SIGCHI symphosium on Engineering interactive computing systems, pp.43-48, 2013

[16] M. González-García, L. Moreno, P. Martínez, Adaptation rules for Accessible Media Player Interface, Proceedings of the XV International Conference on Human Computer Ineraction, vol: ACM, Article 5, 2014

[17] R. Contreras, A. Zisman, Identifying, Modifying, Creating, and Removing Monitor Rules for Service Oriented Computing, Proceedings of the $3^{\text {rd }}$ International Workshop on Principles of Engineering Service-Oriented Systems, pp.4349, 2011.

[18] S. Franke and T. Neumuth, Rule-based medical device adaptation for the digital operating room, Proceedings of EMBC, pp.1733-1736, 2015.

[19] S. W. Dietrich, R. Patil, A. Sundermier, S. D. Urban, Component adaptation for event-based application integration using active rules, The Journal of Systems and Software vol. 79, pp. 1725-1734, 2006.

[20] H. Li, D. Hu, T. Hao, L. Wenyin, X. Chen, Adaptation Rule Learning for Case-Based Reasoning, Concurrency and Computation Practice and Experience, vol. 21(5), pp. 44-49, 2007.

[21] K. Karthik, Key Search and Adaptation based on Association Rules for Backward Secrecy, Proceedings of International Workshop on Information Forensics and Security (WIFS), 2015.

[22] S. Lemouzy, V. Camps, P. Glize, Towards a self-organising mechanism for learning adaptive decision-making rules, In: Proceedings of International Conference on Web Intelligence and Intelligent Agent Technology, pp.616-620, 2008.

[23] I. Lanese, A. Bucchiarone, and F. Montesi, A Framework for Rule-Based Dynamic Adaptation, In: Wirsing M., Hofmann M., Rauschmayer A. (eds) Trustworthly Global Computing. TGC 2010. Lecture Notes in Computer Science, vol. 6084. Springer, Berlin, Heidelberg, 2010.

[24] M. Zeeshan, S. A. Khan, A novel algorithm for link adaptation using fuzzy rule based system for wideband networking waveform of SDR, International Journal of Electronics and Communications (AEÜ), vol.69, pp. 13661373,2015

[25] A. Abraham, Rule-based Expert Systems, Handbook of Measuring System Design, edited by Peter H. Sydenham and Richard Thorn, 2005.
[26] R. Contreras, A. Zisman, A. Marconi and M.Pistore, PRadapt: A Framework for Dynamic Monitoring of Adaptable Service-Based Systems, Proceedings of ICSE Workshop on Principles of Engineering Service Oriented Systetms, pp.50-57 2012

[27] Y. Liu, W. Zhang and W. Jiao, A Generative Genetic Algorithm for Evolving Adaptation Rules of Software Systems, In: Proceedings of the $8^{\text {th }}$ Asia-Pacific Symphosium on Internetware, pp.103 - 107, 2016.

[28] V. Kumar, A. Fensel and P. Fröhlich, Context Based Adaptation of Semantic Rules in Smart Buildings, Proceedings of International Conference on Information Integration and Web-based Applications \& Services, 2013.

[29] H. Wang, R. Mehta, S. Supakkul, L. Chung, Rule-based Context-aware Adaptation Using a Goal-Oriented Ontology, Proceedings of the 2011 International Workshop on Situation Activity \& Goal Awareness, pp.67-76, 2011.

[30] P. Haitao,,L. Jinjiao, L. Fasheng, Mobile Device Adaptation Based on Business Rules, Proceedings of the Joint Conferences on Pervasive Computing (JCPC), pp. 675-678, 2009

[31] L.Bradji, M. Boufaida, A Rule Management System for Knowledge Based Data Cleaning, Intelligent Information Management, vol 3, pp. 230-239, 2011.

[32] G. Ge, E. J. Whitehead, Jr. (Advisor), Automatic Generation of Rule-based Software Configuration Management Systems, Proceedings of $27^{\text {th }}$ International Conference on Software Engineering, 2005.

[33] T. Zhao, The Generation and Evolution of Adaptation Rules in Requirements Driven Self-adaptive Systems, Proceedings of the Requirements Engineering Conference (RE), pp. 456461, 2016.

[34] Q. Wang, Towards a Rule Model for Self-adaptive Software, ACM SIGSOFT Software Engineering Notes, pp. 1-5, 2005.

[35] J. Li, H. P. H. Shum, X. Fu, G. Sexton, L. Yang, Experiencebased Rule Base Generation and Adaptation for Fuzzy Interpolation, Proceedings of 2016 IEEE International Conference on Fuzzy Systems (FUZZ-IEEE), 2016.

[36] M. Soui, A. Abdelbaki, M. Kessentin and K. Ghedira, Improving Adaptation rules Quality Using Genetic Programming, In: Procedia Computer Science, vol. 21 pp. 274 - 281, 2013. 